\title{
Association of a Multigenetic Pro-Inflammatory Profile with Ischaemic Stroke
}

Frederick Palm ${ }^{\mathrm{a}}$ Annette Aigner ${ }^{\mathrm{b}}$ Pirkko Johanna Pussinen ${ }^{\mathrm{c}}$

Christian Urbanek $^{d}$ Florian Buggle $^{d}$ Anton Safer ${ }^{\text {e Heiko Becher }}{ }^{f}$

Armin J. Grau ${ }^{\text {d }}$

${ }^{a}$ Department of Neurology, Helios Klinikum Schleswig, Schleswig, Germany; ${ }^{b}$ Institute of Biometry and Clinical Epidemiology, Charité, Universitätsmedizin Berlin, Berlin, Germany; ${ }^{C}$ Oral and Maxillofacial Diseases, University of Helsinki, Helsinki, Finland; ${ }^{d}$ Department of Neurology, Klinikum Ludwigshafen, Ludwigshafen, Germany; ${ }^{\mathrm{e}}$ Institute of Global Health, University of Heidelberg, Heidelberg, Germany; ${ }^{f}$ Institute of Medical Biometry and Epidemiology, University Medical Center Hamburg-Eppendorf, Hamburg, Germany

\section{Keywords}

Ischemic stroke · Inflammation · Gene score · Single

nucleotide polymorphism

\begin{abstract}
Objective: A multigenetic pro-inflammatory profile may increase stroke risk. We investigated whether a higher number of pro-inflammatory genetic variants are associated with ischaemic stroke risk and whether other risk factors further elevate this risk. Methods: In a case-control study with 470 ischaemic stroke patients (cases) and 807 population controls, we investigated 23 haplotypes or alleles in 16 inflammatory genes (interleukin [IL]1A, IL1B, IL1 receptor antagonist, IL6, IL6 receptor, IL10, tumour necrosis factor-a; C-C motif chemokine ligand 2, C-C motif chemokine receptor 5, C-reactive protein (CRP), intercellular adhesion molecule 1, transforming growth factor $\beta 1$, E-Selectin, selenoprotein $\mathrm{S}$, cluster determinant 14, histone deacetylase 9 [HDAC9]). We constructed an extended gene score (EGS) as the sum of all individual risk alleles and analysed its effect on stroke, just as
\end{abstract}

its association and interaction with cardiovascular risk factors and infectious scores (IgG antibodies against 5 respectively $\lg \mathrm{A}$ antibodies against 4 microbial antigens). Results: Cases were less likely to carry the minor allele of IL10 rs1800872 and more likely to carry the HDAC9 allele rs11984041 and the pro-inflammatory haplotype of CRP, although the latter was not statistically significant in our study. Overall, cases tended to have more pro-inflammatory alleles and haplotypes than controls (mean \pm SD $13.25 \pm 2.25$ and $13.04 \pm 2.41$, respectively). However, the EGS only slightly and not significantly increased the risk of stroke (OR 1.04, 95\% Cl 0.99-1.09). Its effect was neither associated with included risk factors nor with IgA and IgG infectious scores, and we found no significant interaction effects. Conclusion: A more pro-inflammatory genetic profile might increase stroke risk to some extent. This potential effect is most likely independent of established cardiovascular risk factors and the infectious burden of an individual.

(c) 2020 S. Karger AG, Basel

F.P. and A.A. contributed equally to this work.

\section{KARGER}

(c) 2020 S. Karger AG, Basel

karger@karger.com

www.karger.com/ced
Frederick Palm, MD, PhD

Department of Neurology

Helios Klinikum Schleswig

St. Jürgener Strasse, 1-3, DE-24837 Schleswig (Germany)

E-Mail frederick.palm@helios-gesundheit.de 


\section{Introduction}

Several inflammatory parameters such as C-reactive protein, leukocyte count and fibrinogen, as well as acute infectious diseases are associated with the risk of stroke [1-4]. The inflammatory response to various stimuli is under complex genetic control. Genetic risk factors seem to interact in a highly complex biological process [5]. Currently, almost 700 gene products are known to be associated with the inflammatory response [6]. In most inflammatory genes, common sequence variants (polymorphisms) occur, which modify their expression or their activity. Pro-inflammatory alleles of many inflammatory genes (e.g., C-C motif chemokine receptor 5 [CCR5], C-reactive protein [CRP], interleukin [IL]6, IL1A, IL1 receptor antagonist [IL1RN], selenoprotein S1 [SEPS1], and transforming growth factor $\beta 1$ [TGFB1]) have previously been studied in stroke patients [1, 2, 7], and recent genome-wide association studies (GWAS) identified associations of variants in some inflammatory genes (LIPA, SMAD3, histone deacetylase 9 [HDAC9]) with coronary artery disease or ischemic stroke [8].

As genetic factors modify the strength and the sensitivity of the inflammatory response, it is hypothesised that the risk of vascular diseases including stroke increases with the number of pro-inflammatory polymorphisms of an individual. The phenotypic effect size of isolated common genetic variants is usually small, but combined effects of pro-inflammatory genotypes from single or multiple inflammatory pathways may considerably enhance the risk. Genetic scores have previously been proposed, such as the inflammatory gene score (IGS) [9] and the inflammatory load score [10].

The case-control study of genetic, socioeconomic and infectious factors in ischaemic stroke study was set out to explore genetic, socioeconomic and infectious risk factors for stroke and their interdependence. Previous publications on the same study focused on the latter 2 aspects $[3,4]$. Here, we assess the association of ischaemic stroke with individual pro-inflammatory gene variants. Additionally, we propose a new extended inflammatory gene score (EGS) based on 16 inflammatory gene variants previously found associated with stroke or cardiovascular disease and/or with the inflammatory response and evaluate its association with ischaemic stroke risk. Furthermore, we explore the associations between the EGS and established stroke risk factors and infectious scores, just as their interaction.

Multigenetic Pro-inflammatory Profile in Ischemic Stroke

\section{Methods}

Inclusion and Exclusion Criteria

The study population was described in a previous paper [3]. Cases were first-ever ischaemic stroke patients aged 18-80 years living in the city of Ludwigshafen am Rhein in South-West Germany. Age and sex frequency-matched controls were randomly selected from the general population of Ludwigshafen. All cases received neuroimaging excluding cerebral haemorrhage. Cases with transient ischaemic attack were excluded, just as those with intracerebral, subdural or subarachnoid haemorrhage and those with ischaemic stroke due to cerebral trauma or brain malignancy. Both cases and controls were excluded in case of previous stroke, myocardial infarction within 90 days, dementia, severe aphasia or any other communication barrier or severe disability. Cardiovascular risk factors were defined according to current national and international guidelines and have been described in detail [3]. All subjects gave written informed consent to participate in the study. The study was approved by the Ethics Committee of the Landesärztekammer Rheinland-Pfalz (837.333.05[4991]).

\section{Genetic Samples}

Fresh whole blood samples $(2 \times 5 \mathrm{~mL})$ from all cases and controls were drawn, labelled with a pseudonymised code and sent from Ludwigshafen to the laboratory of molecular genetics of the University of Heidelberg Neurology Department, where the analysis of genetic samples was performed. One aliquot of the blood was stored at $-20^{\circ} \mathrm{C}$. From the other aliquot, DNA was isolated by sodium dodecyl sulphate-proteinase-K digestion, phenol-chloroform extraction and ethanol precipitation. DNA was re-dissolved in distilled water, quantified by UV spectrometry, diluted at a concentration of $50-200 \mu \mathrm{g} / \mathrm{mL}$ and stored at $-20^{\circ} \mathrm{C}$.

For genotyping of all but 2 genetic variants, sequences flanking the single nucleotide polymorphisms (SNP) were amplified by polymerase chain reaction, followed by restriction fragment length polymorphism analysis. CCR5 - rs333, a 32 base-pair deletion, was genotyped directly by $2 \%$ agarose gel electrophoresis, and the IL-6 promotor haplotypes were assessed by Sanger sequencing of the amplified fragment.

Two scientists carefully checked restriction fragment length polymorphism results to detect incomplete restriction enzyme digestion. After quality review, unsuccessful genotyping was repeated. Hardy-Weinberg equilibrium was analysed for each genotyped variant. SNPs known to be in linkage disequilibrium were analysed with the haploview software to confirm linkage disequilibrium as an additional check.

\section{Extended Gene Score}

We selected 23 alleles or haplotypes of 16 inflammatory genes on the basis of the following criteria: The gene variant (i) was found to be associated either with the risk of ischemic stroke or myocardial infarction in previous studies; (ii) belongs to the group of inflammatory genes or (iii) possesses alleles or haplotypes which were reported to be associated with a higher or lower inflammatory response. Non-inflammatory genes and genes without reported association with above vascular diseases were not considered. The following variants were selected: IL1A rs1894399 [11], IL1B rs16944 [12], IL1RN rs380092 [12], IL6 pro-inflammatory haplotype [13], IL6 receptor (IL6R) rs2228145 [14], IL10 rs1800872 [15], tumour necrosis factor-a (TNF) rs1800629 [16], C-C motif che-

Cerebrovasc Dis 2020;49:170-176 
Table 1. Genotypes of single polymorphisms in controls and cases

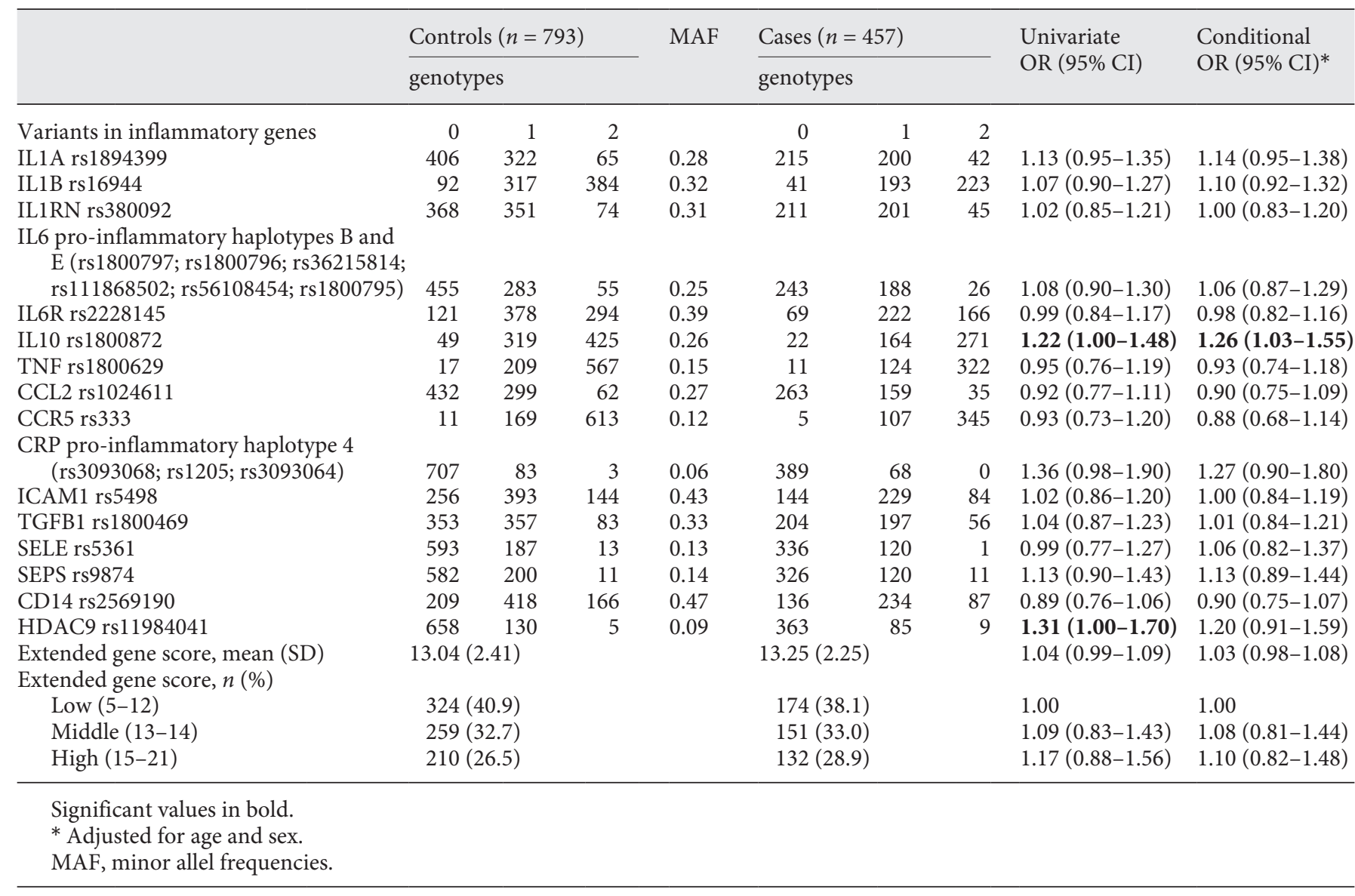

mokine ligand 2 rs1024611 [17], CCR5 rs333 [17], CRP pro-inflammatory haplotype [18], intercellular adhesion molecule 1 rs5498 [5], TGFB1 rs1800469 [19], E-Selectin rs5361 [20], SEPS rs9874 [21], cluster determinant (CD) 14 rs2569190 [22, 23] and HDAC9 rs11984041 [24]. Haplotype analysis was performed for CRP (3 SNPs) and IL6 (6 SNPs). Minor allele frequencies are reported in Table 1. In addition to investigating the individual SNPs or haplotypes, we constructed an EGS based on all risk alleles of the 16 genes. In 5 genes (IL1B, IL6R, IL10, CCR5 and TNF), the frequent allele was counted as the pro-inflammatory variant and thus as the risk allele, from the other 11 genes the rare allele was considered as the risk allele. For each risk gene, a subject without risk allele was scored as ' 0 ', a subject heterogeneous for the risk allele as ' 1 ', and a subject homozygous for the respective risk allele as ' 2 '. The EGS is the sum of all risk genes in our study.

Established Cardiovascular Risk Factors and Infectious Scores

We included hypertension, diabetes, hypercholesterolemia, atrial fibrillation and current smoking as cardiovascular risk factors, defined according to international guidelines as previously described [3].

Infectious scores were constructed based on seropositivity to Aggregatibacter actinomycetemcomitans and Porphyromonas gingivalis, Chlamydia pneumonia, Mycoplasma pneumoniae (IgA and
IgG each) and CagA-positive Helicobacter pylori (IgG) [4]. This resulted in one score based on IgA seropositivity and one on IgG seropositivity. In contrast to our previous publication [4], we used these scores as continuous measures.

\section{Statistical Analysis}

We present frequencies and distributions of individual gene variants and the EGS. The EGS was primarily analysed as a continuous measure, but also categorised into approximate tertiles based on its distribution in the controls.

For the main analyses, we present OR estimates along with 95\% $\mathrm{CI}$ based on unconditional and conditional univariate logistic regression, the latter conditioned on sex and 2-year age groups.

To assess the association of EGS with established cardiovascular risk factors and the infectious scores, we first account for them in a conditional logistic regression model, and second, test their interaction effect with EGS individually. We further explore the effect of EGS within subsets by median age and TOAST stroke subtypes.

As all analyses are of exploratory nature, we did not adjust for multiple testing. We performed complete-case analyses, thereby excluding 13 cases and 14 controls from all analyses due to missing data on genetic variants or cardiovascular risk factors.

The statistical software R was used for all analyses [25]. 


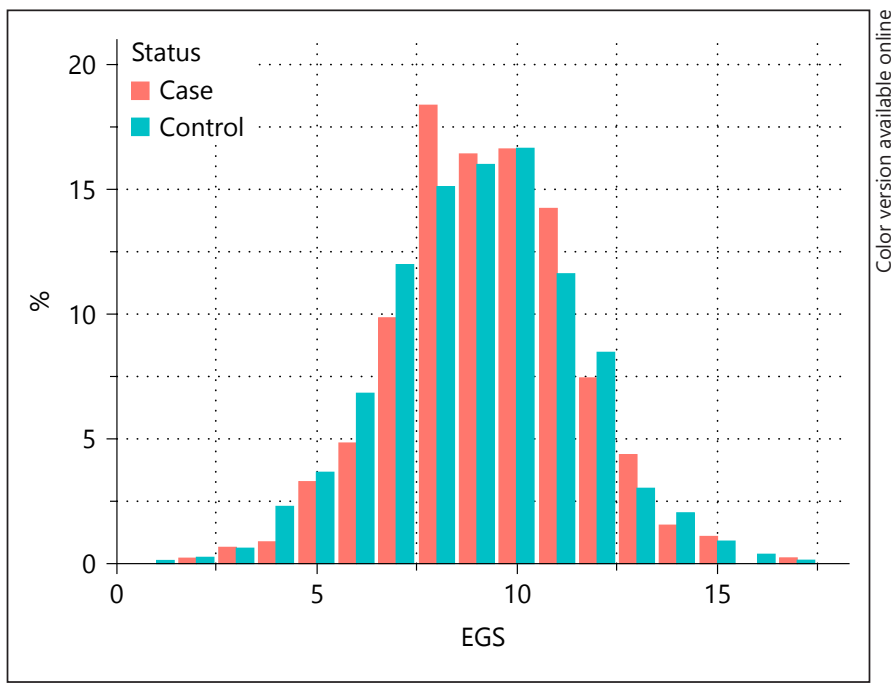

Fig. 1. Relative distribution of the EGS by case-control status. EGS, extended gene score.

\section{Results}

The analyses included 457 cases (183 women and 274 men) and 793 controls ( 327 women, 466 men), with a median age of 69 years. Cases showed slightly more pro-inflammatory alleles and haplotypes (mean \pm SD $13.25 \pm$ $2.25)$ compared to controls $(13.04 \pm 2.41)$. Figure 1 visualizes the distribution of score values in cases and controls.

The lower frequency of the minor anti-inflammatory allele of IL10 rs1800872 significantly increased the odds of ischaemic stroke by $26 \%$ (conditional OR 1.26, 95\% CI 1.03-1.55). The higher frequency of the minor allele of HDAC9 rs11984041 (conditional OR 1.20, 95\% CI 0.911.59 ) and of the pro-inflammatory haplotype of CRP (conditional OR 1.27, 95\% CI 0.90-1.80) were associated with increased stroke risk to a similar extent although with a less precise estimation of these effects, rendering them not statistically significant (Table 1, Fig. 2).

The unconditional logistic regression for the EGS showed that an additional pro-inflammatory variant increased the odds of stroke by $4 \%$ (OR 1.04, 95\% CI 0.991.09), although this effect was not significantly different from no increase. Results did not change substantially after adjustment for age and sex (OR 1.03, 95\% CI 0.98-1.08).

Adjusting for established cardiovascular risk factors did not change the effect of EGS, neither did the adjustment for infectious scores (Fig. 2). Without further adjustment, the IgA infectious score was individually associated with the risk of stroke (OR 1.16, 95\% CI 1.00-1.35), whereas the IgG score was not (OR 1.00, 95\% CI 0.90-1.12).

Multigenetic Pro-inflammatory Profile in Ischemic Stroke
Based on individual conditional logistic regression, there was neither a significant statistical interaction between any cardiovascular risk factor and EGS ( $p$ value of interaction for hypertension: 0.44 ; diabetes: 0.38 ; hypercholesterolemia: 0.87; atrial fibrillation: 0.60; smoking: 0.63 ) nor between the infectious scores and EGS (IgA score: 0.70 ; IgG score: 0.061 ).

There was no notable difference in the unconditional effect of EGS for age at stroke onset. The OR in the group with early age onset ( $<70$ years: OR $1.04,95 \%$ CI $0.97-$ 1.12 ) was the same as in the later onset group ( $\geq 70$ years: OR 1.03, 95\% CI 0.97-1.11). There were also no relevant differences in the effect of EGS comparing stroke aetiology, according to the TOAST stroke subtypes (large vessel disease $(n=158)$ : OR 1.00, 95\% CI 0.94-1.08; Cardioembolism $(n=103)$ : OR 1.06, 95\% CI 0.98-1.16; small vessel disease ( $n=137)$ : OR $1.06,95 \%$ CI $0.98-1.14$; other $(n=59)$ : OR $1.03,95 \%$ CI $0.93-1.15)$.

\section{Discussion}

\section{Summary}

Our study assessed whether a more pro-inflammatory genetic profile increases the risk of stroke. Furthermore, we hypothesised that established cardiovascular risk factors, just as a higher infectious burden as measured with seropositivity to several microbial agents would be associated with the pro-inflammatory gene load, potentially interacting with it and as such further increasing stroke risk. Using the EGS, a genetic score based on 23 variants in 16 inflammatory genes, we found a more pro-inflammatory profile being positively associated with the risk of stroke, however, with a small effect which was not significantly different from no effect (OR 1.04, 95\% CI 0.99-1.09). Furthermore, we could neither identify any association or interaction between the genetic score and the included cardiovascular risk factors nor the infectious burden based on 5 microbial agents. Three polymorphisms previously found associated with stroke showed a relevant effect on ischemic stroke in our study, with OR estimates above 1.20 - IL10 rs1800872, HDAC9 rs11984041 and CRP, although the latter 2 were not statistically significant in the unconditional analysis.

\section{Comparison with Previous Studies}

The association of HDAC9 rs11984041 with ischaemic stroke was established in a large-GWAS including several study populations achieving a combined p-value of $1.87 \times 10^{-11}$. The OR was $1.42(95 \%$ CI $1.28-1.57)$ [26], 
Fig. 2. OR estimates of individual variants and the EGS based on conditional logistic regression (conditioned on sex and 2-year age groups), EGS results furthermore adjusted for infectious scores and cardiovascular risk factors. IL, interleukin; IL1RN, IL1 receptor antagonist; IL6R, IL6 receptor; TNF, tumour necrosis factor-a; CCL2, C-C motif chemokine ligand 2; CCR5, C-C motif chemokine receptor 5; CRP, C-reactive protein; ICAM, intercellular adhesion molecule 1; TGFB, transforming growth factor $\beta 1$; SELE, E-Selectin; SEPS, selenoprotein S; CD14, cluster determinant 14, HDAC9, histone deacetylase 9; EGS, extended gene score.

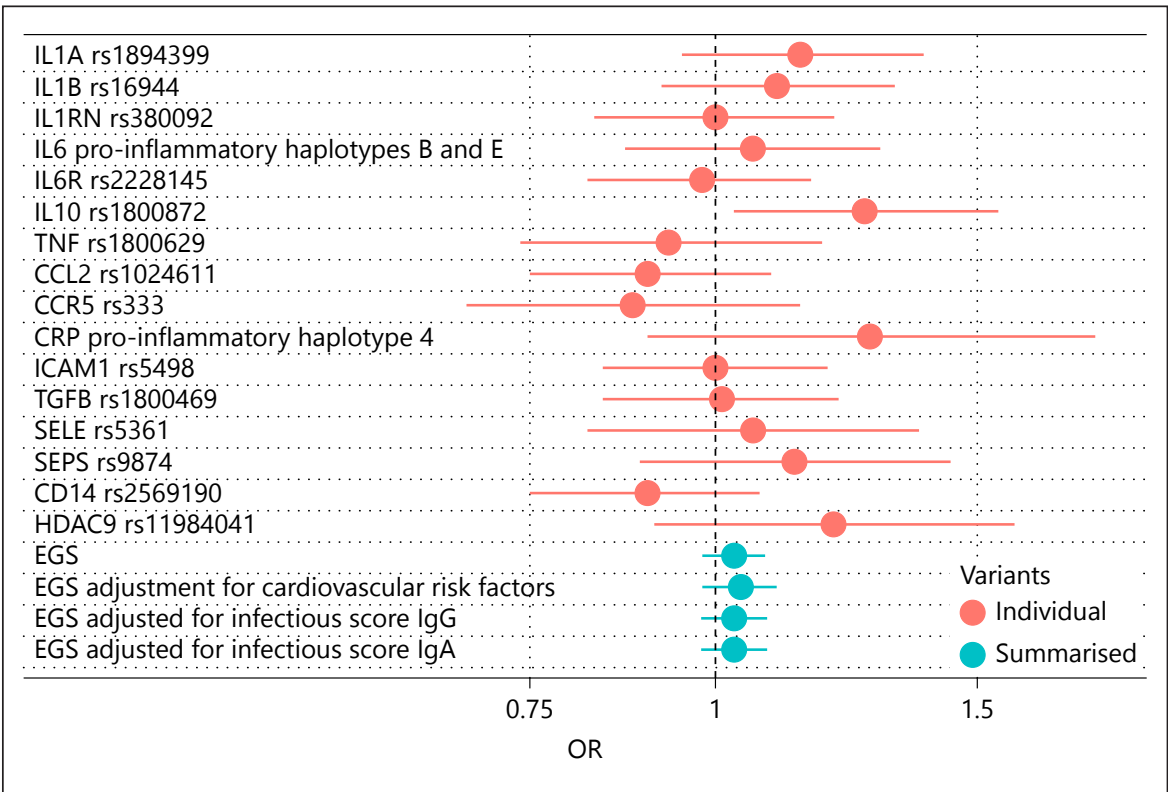

which is in line with our finding of an unconditional OR of 1.32 (95\% CI 1.00-1.70). HDAC9 belongs to a large family of genes which are responsible for deacetylation of histones and thus for regulation of chromatin structure and gene transcription [24]. Beyond other roles, it is an important regulator of inflammatory factor expression including IL-6 signalling and was thus included in the EGS. IL10 is a pleiotropic cytokine with predominantly anti-inflammatory effects, potentially protective against atherosclerosis. IL10 rs1800872 is a promotor polymorphism supposed to be associated with lower IL10 plasma levels [15]. The association of IL10 rs 1800872 with ischaemic stroke which we found in our sample was previously observed in a Chinese population [15], but large GWAS of ischemic stroke patients and healthy controls did not report a significant association with the IL10 polymorphism.

A few other SNPs were associated with a trend towards higher stroke risk in our study, for example, IL1A rs1894399, CRP haplotype 4, IL1B rs16944, or SEPS rs9874. In several other SNPs, the effect was completely neutral or even associated with a trend towards reduced stroke risk (e.g., CD14 rs2569190). Recent studies and meta-analyses investigated the role of inflammatory polymorphisms on stroke risk. Different to our results, intercellular adhesion molecule 1 rs5498 was found to be associated with ischaemic stroke risk in Caucasians but not in Asians in a meta-analysis [5]. In contrast to some previous studies and in line with the results presented here, a recent meta-analysis did not detect a significantly in- creased risk of stroke in carriers of ILA rs1894399 and CD14 rs2569190 [19]. The rare allele of TNF rs1800629 was protective against stroke in Asian, but not in Caucasian populations according to a recent meta-analysis [16], in line with the neutral result in our population. In Chinese populations, TGFB1 rs1800469 and E-Selectin rs5361 were associated with stroke risk in contrast to our neutral result in a Caucasian population $[19,20]$.

\section{Strengths and Limitations}

It is the advantage of our study that we investigated a larger number of SNPs than previous similar studies. However, our study has several methodological limitations. First, the sample size of our case-control study was not estimated with the aim of detecting significant effects of the presented analyses. The assumptions for the power estimations related to the association between stroke and socioeconomic risk factors, but not for the genetic analysis. Therefore, these results must be considered to be of exploratory nature. Second, as we tested multiple gene variants and did not adjust for multiple testing, the few significant findings would be expected for purely statistical reasons and as such might be without any biological value - despite being in line with previous findings. Third, the number of microbial agents investigated in the infectious score was limited and a more extended number of viruses and bacteria might have yielded different results. Furthermore, including clinically diagnosed chronic infectious diseases instead of serological data might have resulted in more reliable findings. 


\section{Conclusion}

In our study population, the pro-inflammatory genetic burden based on 23 genetic variants in 16 genes in cases was slightly higher than in controls, but the difference was small and not significant. Thus, our results do not contradict, but also do not reliably support our primary hypothesis that carriers of an elevated number of proinflammatory alleles have an increased risk of ischaemic stroke. Furthermore, we did not have any findings to confirm the hypothesis that subjects with a higher infectious burden are particularly at increased risk of ischaemic stroke if they additionally carry a higher number of proinflammatory gene variants.

In the future, appropriately powered studies investigating a much larger number of inflammatory gene variants will be required to answer the question whether a more pro-inflammatory genetic profile is associated with increased risk of ischaemic stroke.

\section{Acknowledgement}

We gratefully acknowledge the help of Caspar Grond-Ginsbach $(\mathrm{PhD})$, Dr. Marie-Luise Arnold and Inge Werner in the genetic analyses in this study.

\section{Statement of Ethics}

All authors state that published research is in compliance with the guidelines for human studies. Research was conducted ethically in accordance with the World Medical Association Declaration of Helsinki.

\section{Disclosure Statement}

The authors have no conflicts of interest to declare.

\section{Funding Sources}

This work was supported by the German Research Foundation (BE 2056/5-1, GR 1102/5-1, GR 1687/2-1), the Academy of Finland (Grant number 1266053) and the Finnish Dental Association Apollonia.

\section{Author Contributions}

A.J.G., H.B., F.P. and P.J.P. were responsible for study concept and design. C.U., F.P., and F.B. were responsible for data acquisition. A.A., A.S. and H.B. mainly contributed to statistical analysis. F.P. and A.A. wrote the manuscript. F.B., A.S., H.B., P.J.P., C.U. and A.J.G. critically revised and edited the manuscript for important intellectual content.

\section{References}

1 Lozano ML, González-Conejero R, Corral J, Rivera J, Iniesta JA, Martinez C, et al. Polymorphisms of P-selectin glycoprotein ligand-1 are associated with neutrophil-platelet adhesion and with ischaemic cerebrovascular disease. Br J Haematol. 2001 Dec;115(4): 969-76.

2 Revilla M, Obach V, Cervera A, Dávalos A, Castillo J, Chamorro A. A -174G/C polymorphism of the interleukin- 6 gene in patients with lacunar infarction. Neurosci Lett. 2002 May;324(1):29-32.

3 Becher H, Palm F, Aigner A, Safer A, Urbanek $\mathrm{C}$, Buggle F, et al. Socioeconomic conditions in childhood, adolescence, and adulthood and the risk of ischemic stroke. Stroke. 2016 Jan; 47(1):173-9.

4 Palm F, Pussinen PJ, Aigner A, Becher H, Buggle F, Bauer MF, et al. Association between infectious burden, socioeconomic status, and ischemic stroke. Atherosclerosis. 2016 Nov;254:117-23.

5 Zhang MJ, Zhang M, Yin YW, Li BH, Liu Y, Liao SQ, et al. Association between intercellular adhesion molecule-1 gene K469E polymorphism and the risk of stroke in a Chinese population: a meta-analysis. Int J Neurosci. 2015 Mar;125(3):175-85.
6 Bult CJ, Blake JA, Smith CL, Kadin JA, Richardson JE; Mouse Genome Database Group. Mouse genome database (MGD) 2019. Nucleic Acids Res. 2019 Jan;47(D1):D801-6.

7 Lindsberg PJ, Grau AJ. Inflammation and infections as risk factors for ischemic stroke. Stroke. 2003 Oct;34(10):2518-32.

8 Grond-Ginsbach C, Hummel M, Wiest T, Horstmann S, Pfleger K, Hergenhahn M, et al. Gene expression in human peripheral blood mononuclear cells upon acute ischemic stroke. J Neurol. 2008 May;255(5):72331.

9 Flex A, Gaetani E, Papaleo P, Straface G, Proia AS, Pecorini G, et al. Proinflammatory genetic profiles in subjects with history of ischemic stroke. Stroke. 2004 Oct;35(10): $2270-5$.

10 Jerrard-Dunne P, Sitzer M, Risley P, Buehler A, von Kegler S, Markus HS. Inflammatory gene load is associated with enhanced inflammation and early carotid atherosclerosis in smokers. Stroke. 2004 Nov;35(11):243843.

11 Banerjee I, Gupta V, Ahmed T, Faizaan $\mathrm{M}$, Agarwal P, Ganesh S. Inflammatory system gene polymorphism and the risk of stroke: a case-control study in an Indian population. Brain Res Bull. 2008 Jan;75(1): 158-65.

12 Olsson S, Holmegaard L, Jood K, Sjögren M, Engström G, Lövkvist $H$, et al. Genetic variation within the interleukin-1 gene cluster and ischemic stroke. Stroke. 2012 Sep;43(9):227882.

13 Acalovschi D, Wiest T, Hartmann M, Farahmi M, Mansmann U, Auffarth GU, et al. Multiple levels of regulation of the interleukin-6 system in stroke. Stroke. 2003 Aug;34(8): 1864-9.

14 Miwa K, Okazaki S, Sakaguchi M, Mochizuki H, Kitagawa K. Interleukin-6, interleukin-6 receptor gene variant, small-vessel disease and incident dementia. Eur J Neurol. 2016 Mar;23(3):656-63.

15 Xie G, Myint PK, Zaman MJ, Li Y, Zhao L, Shi $\mathrm{P}$, et al. Relationship of serum interleukin-10 and its genetic variations with ischemic stroke in a Chinese general population. PLoS One. 2013 Sep;8(9):e74126.

16 Kumar P, Misra S, Kumar A, Pandit AK, Chakravarty K, Prasad K. Association between Tumor Necrosis Factor- $\alpha$ (-238G/A and -308G/A) Gene Polymorphisms and Risk of Ischemic Stroke: A Meta-Analysis. Pulse (Basel). 2016 Apr;3(3-4):217-28. 
17 Afzal AR, Kiechl S, Daryani YP, Weerasinghe A, Zhang Y, Reindl M, et al. Common CCR5-del32 frameshift mutation associated with serum levels of inflammatory markers and cardiovascular disease risk in the Bruneck population. Stroke. 2008 Jul;39(7): 1972-8.

18 den Hertog HM, van den Herik EG, Dippel DW, Koudstaal PJ, de Maat MP. Variation in the $\mathrm{C}$-reactive protein gene is associated with serum levels of CRP in patients with acute ischemic stroke. Cerebrovasc Dis. 2010;29(4): $372-5$.

19 Tao HM, Chen GZ, Cheng GP, Shan XY. The haplotype of the TGF $\beta 1$ gene associated with cerebral infarction in Chinese. Can J Neurol Sci. 2012 Sep;39(5):626-31.
20 Zhao DX, Feng J, Cong SY, Zhang W. Association of E-selectin gene polymorphisms with ischemic stroke in a Chinese Han population. J Neurosci Res. 2012 Sep;90(9):1782-7.

21 Curran JE, Jowett JB, Elliott KS, Gao Y, Gluschenko K, Wang J, et al. Genetic variation in selenoprotein S influences inflammatory response. Nat Genet. 2005 Nov;37(11): 1234-41.

22 Lichy C, Meiser H, Grond-Ginsbach C, Buggle F, Dörfer C, Grau A. Lipopolysaccharide receptor CD14 polymorphism and risk of stroke in a South-German population. J Neurol. 2002 Jul;249(7):821-3.

23 Misra S, Kumar P, Kumar A, Sagar R, Chakravarty $\mathrm{K}$, Prasad K. Genetic association between inflammatory genes (IL-1 $\alpha$, CD14,
LGALS2, PSMA6) and risk of ischemic stroke: A meta-analysis. Meta Gene. 2016 Jan; 8:21-9.

24 Bellenguez C, Bevan S, Gschwendtner A, Spencer CC, Burgess AI, Pirinen M, et al.; International Stroke Genetics Consortium (ISGC); Wellcome Trust Case Control Consortium 2 (WTCCC2). Genome-wide association study identifies a variant in HDAC9 associated with large vessel ischemic stroke. Nat Genet. 2012 Feb;44(3):328-33.

25 R Core Team. R: A language and environment for statistical computing. Vienna, Austria: R Foundation for Statistical Computing; 2019.

26 Markus HS. WellcomeTrust genome-wide association study of ischemic stroke. Stroke. 2013 Jun;44(6 Suppl 1):S20-2. 